\title{
RELATO DE EXPERIÊNCIA: PRÁTICAS DE ENGENHARIA BIOMÉDICA VOLTADAS A SEGURANÇA DE PACIENTES E PROFISSIONAIS DE SAÚDE ${ }^{1}$
}

\author{
EXPERIENCE REPORT: BIOMEDICAL ENGINEERING PRACTICES ON \\ PATIENT AND HEALTH PROFESSIONALS SAFETY
}

\section{Helena Oliveira Bertolino², Karla Cristiane Oliveira Silva ${ }^{3}$, Fernando Mariano Bayer ${ }^{4}$, Leandro Welter Caetano ${ }^{5}$, Júlia Vaz Schultz ${ }^{6}$, Maria Isabel Veras Orselli ${ }^{7}$, Liliane Alves Pereira ${ }^{8}$ e Luiz Fernando Rodrigues Junior ${ }^{9}$}

\section{RESUMO}

Ao longo dos anos a Engenharia Biomédica vem demonstrando sua importância por meio de soluções tecnológicas para auxiliar profissionais da saúde no combate às doenças. Sua atuação é fundamental no ambiente hospitalar para realizar o gerenciamento das tecnologias em saúde desde a sua aquisição até o descarte. Sendo assim, o primeiro objetivo deste trabalho é realizar o Plano de Gerenciamento das Tecnologias em Saúde em um hospital de Santa Maria, a partir do levantamento do seu parque tecnológico. Com o surgimento da pandemia causada pelo Coronavírus, os profissionais da saúde se tornaram um grupo de risco específico, pois são expostos diretamente ao risco biológico durante o atendimento aos pacientes. Este cenário imprevisível fez com que os hospitais se adaptassem o mais rápido possível dentro das suas condições, manifestando uma deficiência do Setor Saúde em garantir a segurança dos profissionais envolvidos Em razão disso, foi criado um grupo multidisciplinar com objetivo de desenvolver tecnologias para auxiliar e proteger os profissionais da saúde e indiretamente o paciente, de forma gratuita. E, apesar de terem sido criados neste contexto, os dispositivos produzidos serão capazes de continuar contribuindo no trabalho diário dos Estabelecimentos de Assistência à Saúde, protegendo os trabalhadores de outras doenças igualmente transmissíveis e contaminantes.

Palavras-chave: Pandemia, Covid-19, EPI, EPC.

\section{ABSTRACT}

Over the years, Biomedical Engineering has demonstrated its importance through technological solutions to assist health professionals in combating diseases. Its performance is essential in the hospital environment to manage health technologies from acquisition to disposal. Thus, the first objective of this work is to carry out the Health Technology Management Plan in a hospital in Santa Maria, from the survey of its technological park. With the emergence of the pandemic caused by the Coronavirus, health professionals have become a specific risk group, as they are directly exposed to biological risk during patient care. This unpredictable scenario meant that hospitals had to adapt as quickly as possible under their conditions, manifesting a deficiency in the Health Sector in ensuring the safety of the professionals involved. As a result, a multidisciplinary group was

\footnotetext{
${ }^{1}$ Trabalho de Iniciação Científica.

${ }^{2}$ Acadêmica do Curso de Engenharia Biomédica - Universidade Franciscana - UFN. E-mail: helena.bertolino@ufn.edu.br

${ }^{3}$ Colaboradora externa - Hospital Universitário de Santa Maria - HUSM. E-mail: kcos.karla@gmail.com

${ }^{4}$ Colaborador externo - Colégio Técnico Industrial de Santa Maria - CTISM.

${ }^{5}$ Colaborador externo - Engenheiro Biomédico. E-mail: leandro.caetano@gmail.com

${ }^{6}$ Colaboradora externa - Engenheira Biomédica. E-mail: julia.schultz@ufn.edu.br

${ }^{7}$ Pesquisadora - Universidade Franciscana. E-mail: isabel.veras@ufn.edu.br

${ }^{8}$ Co-orientador - Universidade Franciscana - UFN. E-mail: liliane.pereira@ufn.edu.br

${ }^{9}$ Orientador - Universidade Franciscana - UFN. E-mail: luiz.fernando@ufn.edu.br
} 
created with the objective of developing technologies to assist and protect health professionals and, indirectly, the patient, free of charge. And, despite having been created in this context, the devices produced will be able to continue contributing to the daily work on Health Care Establishments, protecting workers from other equally transmissible and contaminating diseases.

Keywords: Pandemic, Covid-19, PPE, CPE.

\section{INTRODUÇÃO}

A Engenharia Biomédica aplica os princípios e práticas científicas da engenharia no contexto da saúde, cujas áreas de atuação são inumeráveis (PEREIRA, 2010). Assim, ao trabalhar em conjunto com outros profissionais, os engenheiros biomédicos auxiliam no combate às doenças, criando, construindo e fornecendo materiais, ferramentas e tecnologias, utilizados tanto para a pesquisa quanto para o diagnóstico e tratamento, ao proporcionar numerosas possibilidades para profissionais da saúde e pacientes (DYRO, 2004). Nesse contexto, conforme normatiza a ANVISA (2010) no que tange o gerenciamento de tecnologias em saúde em estabelecimentos de saúde; os Estabelecimentos de Assistência à Saúde (EAS) devem promover o gerenciamento de tecnologias em saúde utilizadas na prestação dos seus serviços, de modo a garantir rastreabilidade, qualidade, eficácia, efetividade e segurança dessas tecnologias, desde a sua entrada no EAS até o destino final.

Esse gerenciamento de tecnologias é essencial para o funcionamento adequado dos EASs. No entanto, em relação a essa implementação no trabalho cotidiano, pode se tornar desafiador em determinados contextos como nos casos de catástrofes ou pandemias. Em dezembro de 2019, a Organização Mundial da Saúde (OMS) divulgou globalmente a existência do novo vírus SARS-CoV-2, popularmente conhecido como Coronavírus. Pouco tempo depois, milhões de pessoas já haviam sido infectadas, com milhares indo a óbito, após o início de uma grave pandemia mundial. Até 31 de agosto de 2020, o site estatístico Worldometer (2020) havia notificado 25.622.027 de casos positivos para coronavírus, totalizando 854.235 mortes e 6.845.399 casos ativos no mundo. No Brasil, estes números eram de 3.910.901 casos e 121.515 mortes, com 691,652 casos ativos da doença, na data em questão.

Segundo o Ministério da Saúde (2020), a doença causada pelo vírus, nominada de Covid-19, possui sintomas que se assemelham a uma gripe, uma vez que os acometidos relatam mialgia, febre, tosse, dor de garganta e dispneia, dentre outros, podendo haver quadros mais graves chegando à pneumonia severa. Ademais, a Covid-19 pode aumentar o risco de distúrbios cardiovasculares, tais como lesão cardíaca aguda, choque e arritmias, levando ao infarto agudo do miocárdio (FERRARI, 2020).

Um dos maiores obstáculos para o enfrentamento desta pandemia é o alto risco de contaminação do SARS-Cov-2, pois estima-se que um indivíduo infectado pode transmiti-lo para mais 2,5 pessoas, em média. Este fato demanda intensa preocupação, ainda que sua letalidade seja baixa, em torno 0,5\% e $1 \%$ (TEIXEIRA et al., 2020). O desafio se torna ainda maior quando se trata dos profissionais 
da saúde. Para Ribeiro et al. (2020), a exposição direta ao risco biológico durante o atendimento aos pacientes, torna essa classe de trabalhadores um grupo de risco específico. Diante deste cenário inesperado e hostil, percebe-se uma deficiência do Setor Saúde em garantir a segurança dos profissionais envolvidos, além da incerteza relacionada à própria doença e às possibilidades de tratamento.

Sabe-se que uma das estratégias mais essenciais no controle de qualquer tipo de contaminação, incluindo o SARS-Cov-2, entre os profissionais da saúde, é o uso correto de Equipamentos de Proteção Individual (EPIs) durante o atendimento aos pacientes. Estes equipamentos incluem, por exemplo: máscara cirúrgica, máscara PFF2/N95, aventais, óculos de proteção, luvas, protetores faciais etc., além de outros igualmente importantes. Em virtude da pandemia, foram criados protocolos que intensificam seus usos para proteção da equipe, tendo um alto gasto de EPIs, e, consequentemente, ocasionando o aumento do custo e/ou sua escassez no mercado devida à alta demanda.

Portanto, em decorrência da possibilidade da escassez de EPIs nos EASs da cidade de Santa Maria - RS, voluntários de diversas áreas se reuniram para criar a Frente de Desenvolvimento Tecnológico à Saúde (FDTECS), com o objetivo auxiliar os profissionais da saúde no combate à pandemia Covid-19, no intuito de fornecer dispositivos para sua proteção, para tornar seu trabalho mais seguro e para otimizar a assistência ao paciente, sempre de forma gratuita. Nesse sentido, este artigo tem como objetivo descrever um relato de experiência da atuação da engenharia biomédica no contexto hospitalar, por meio da realização do Plano de Gerenciamento das Tecnologias em Saúde em um hospital de Santa Maria, além do relato do desenvolvimento de tecnologias para auxiliar e proteger os profissionais da saúde a partir da FDTECS.

\section{MATERIAIS E MÉTODOS}

As práticas desenvolvidas durante as atividades de extensão foram divididas em duas grandes frentes de trabalho. A primeira foi executada dentro de um hospital da cidade de Santa Maria, sendo essa relacionada diretamente aos Plano de Gerenciamento das Tecnologias em Saúde. A segunda, devido a pandemia, foi executada em conjunto com outros grupos, professores e alunos vinculados a diversas instituições de ensino e hospitais de Santa Maria e ao grupo nacional do Projeto Hígia e está relacionada a construção de Tecnologias para Amparo a Proteção de Profissionais da Saúde.

\section{GERENCIAMENTO DAS TECNOLOGIAS EM SAÚDE NO EAS}

A avaliação do parque tecnológico foi realizada com base no formulário para cadastramento individual de equipamento (CALIL, 1998). Este formulário foi preenchido para cada equipamento individualmente, com auxílio dos profissionais de saúde e técnicos que fazem uso dos mesmos. Também foram elaborados relatórios técnicos sobre o funcionamento dos equipamentos dentro dos EAS. 
Todo a organização foi realizada com o uso de ferramentas de comunicação e compartilhamento de informações, tais como o Teams ${ }^{\circledR}$, Planner $^{\circledR}$ e OneDrive ${ }^{\circledR}$.

Figura 1 - Esquema demonstrando o fluxo de trabalho para o processo de gerenciamento das tecnologias em saúde.

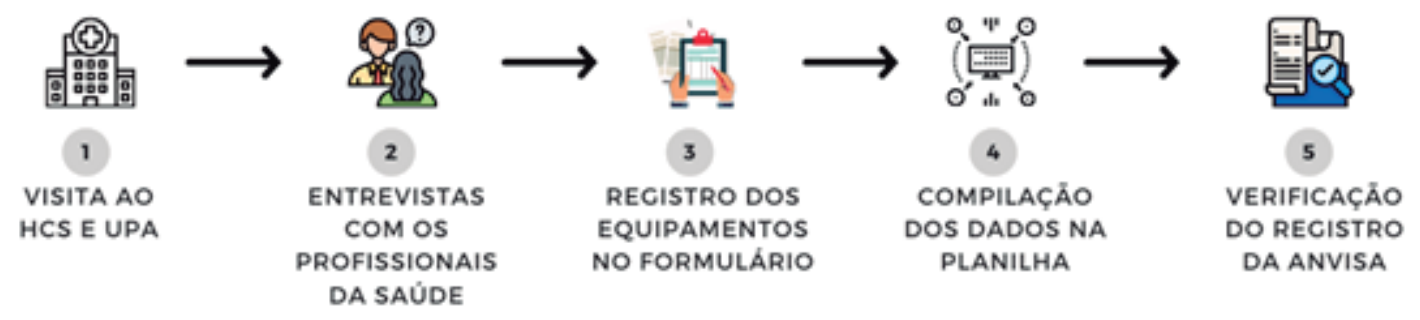

Fonte: Construção do autor

\section{TECNOLOGIAS PARA AMPARO A PROTEÇÃO DE PROFISSIONAIS DA SAÚDE}

Com a pandemia houve a necessidade para o desenvolvimento de tecnologias de proteção individual e coletiva para todos e em especial aos profissionais de saúde. Para tal, foi criado um grupo multidisciplinar e com uso de ferramentas de comunicação virtual e compartilhamento de informações (Teams ${ }^{\circledR}$, OneDrive $^{\circledR}$, WhatsApp $^{\circledR}$ ), esse grupo foi capaz de atuar na cidade de Santa Maria e região para o desenvolvimento, produção e distribuição de EPIs e EPCs. Esse trabalho só foi possível em virtude da RDC 356/2020 que “dispõe, de forma extraordinária e temporária, sobre os requisitos para a fabricação, importação e aquisição de dispositivos médicos identificados como prioritários para uso em serviços de saúde, em virtude da emergência de saúde pública internacional relacionada ao SARS-CoV-2".

Conforme a figura 2A, a partir do surgimento da demanda de protetores faciais, um grupo de voluntários foi reunido, criando a atual FDTECS. Para organização e controle, foi elaborado um formulário de solicitação online, onde os EAS requisitavam os EPIs conforme sua necessidade. Com doações recebidas da comunidade e instituições, foi feita a confecção dos protetores faciais, e, após, doado para cada local.

Devida a percepção da elevada contaminação via contato das mãos ao tocar as maçanetas dos hospitais, conforme a figura 2B, foi pesquisado soluções para tal questão, chegando ao desenvolvimento dos sistemas de maçanetas handsfree. Esses dispositivos foram impressos por manufatura aditiva pelos voluntários, e doados aos hospitais, sendo instalados pela manutenção de cada EAS, de acordo com a sua estratégia.

Baseado no preocupante número de profissionais da saúde que estavam falecendo por conta da Covid-19 (OLIVEIRA, 2020; NEVES, 2020), foi percebida uma alta exposição desses trabalhadores durante os procedimentos de intubação e extubação dos pacientes, mesmo com os EPIs necessários. Segundo a figura 2C, a partir de conversas com os médicos, foram feitas medições das macas 
e camas cirúrgicas para desenvolver as capelas de proteção. Após a confecção e teste do protótipo, foram feitas as correções necessárias e doadas para o hospital.

Figura 2 - Esquemas demonstrando o fluxo de trabalho para o os processos de concepção, produção e entrega dos diferentes produtos deste relato de experiência: (A) protetores faciais (faceshileds);

(B) sistemas de maçanetas handsfree; (C) capelas de intubação e extubação.

(A)

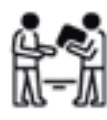

(B)

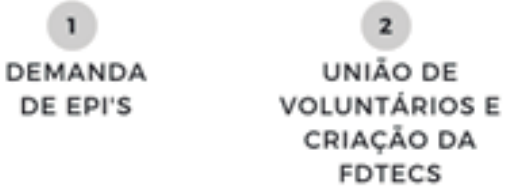

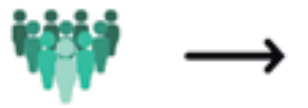

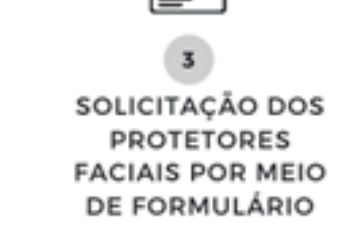

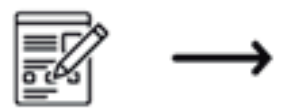
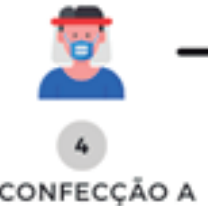

PARTIR DE DOAÇŌES RECEBIDAS

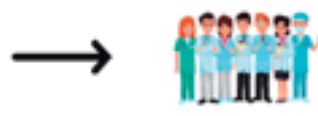

5

DOACCĀO DOS PROTETORES FACIAIS

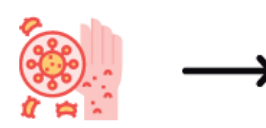

1

PERCEPÇÃO DO ALTO NÚMERO DE CONTAMINAÇÃO VIA CONTATO DAS MÃOS

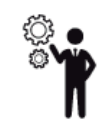

2

BUSCA DE SOLUÇÕES PARA EVITAR O CONTATO DAS MÃOS NAS MAÇANETAS

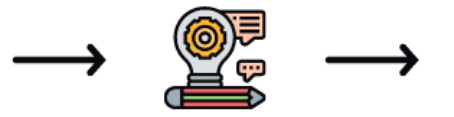

3
DESENVOLVIMENTO IMPRESSÃO 3D 3D DOS DISPOSITIVOS DOS DISPOSITIVOS HANDSFREE

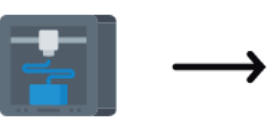

4

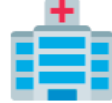

5

ENTREGA DOS SISTEMAS HANDSFREE NOS HOSPITAIS

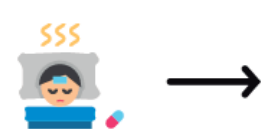

1

(C) CONTAMINAÇÃo DOS MÉDICOS DURANTE OS PROCEDIMENTOS DE INTUBAÇÃO E EXTUBAÇÃO

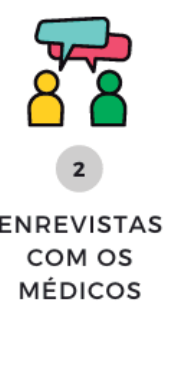

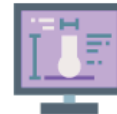

3

MEDIÇÃO DAS CAMAS/MACAS CIRÚRGICAS NO HOSPITAL E ELABORAÇÃO DO MODELO DA CAPELA

Fonte: Construção do autor
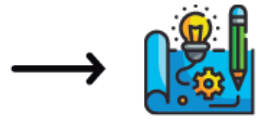

4

TESTE DO PROTÓTIPO E CORREÇÕES NECESSÁRIAS
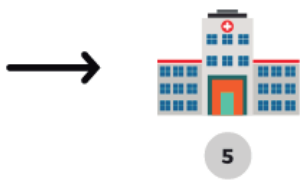

ENTREGA DAS CAPELAS NO HOSPITAL

\section{RESULTADOS E DISCUSSÃO}

\section{GERENCIAMENTO DAS TECNOLOGIAS EM SAÚDE}

O desenvolvimento das práticas de gerenciamento das tecnologias teve início no mês de agosto de 2019 e transcorreu até o início do ano de 2020. Ao longo destes meses foi executado uma varredura em todos os setores do hospital e relacionado o levantamento de cerca de 800 equipamentos médicos. Na figura 3 é apresentado as informações exploradas para realizar a catalogação dos equipamentos médicos presentes no hospital, sendo criada uma planilha no Excel $^{\circledR}$ a partir destas. Esse arquivo foi distribuído a direção do hospital para servir de base ao processo de gerenciamento e rastreabilidade dos equipamentos. 
Figura 3 - Informações obtidas para realização da planilha de catalogação dos equipamentos médicos.

\begin{tabular}{|c|c|}
\hline$\circ$ & Tipo de equipamento (CNES) \\
\hline 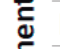 & Nome do equipamento \\
\hline$\frac{\mathrm{c}}{\mathrm{N}}$ & Fabricante \\
\hline 费 & Modelo \\
\hline $\begin{array}{l}0 \\
0\end{array}$ & Ano de fabricação \\
\hline & Tensão/frequência/potência \\
\hline 跑 & Manual online \\
\hline & Manual físico \\
\hline
\end{tabular}

\begin{tabular}{|c|c|}
\hline \multirow{8}{*}{ 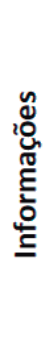 } & Registro da ANVISA - Vencimento \\
\hline & Situação na ANVISA \\
\hline & Classificação de risco \\
\hline & Número de série \\
\hline & Número de Patrimônio \\
\hline & Última calibração \\
\hline & Próxima calibração \\
\hline & Componentes críticos \\
\hline
\end{tabular}

Fonte: Construção do autor

$\frac{\frac{\text { Localização do equipamento }}{\text { Levantamento realizado por }}}{\text { Data do levantamento }}$

Uma das práticas importantes do engenheiro biomédico no ambiente hospitalar é auxiliar na garantia da qualidade e segurança no atendimento dos pacientes. Neste sentido, foram feitas avaliações e elaborados relatórios sobre alguns equipamentos que não estavam em acordo com o preconizado pela RDC 16/2013 ou a RDC 185/2001 que falam da fabricação e uso de equipamentos médicos no território nacional brasileiro. Esses relatórios foram entregues a direção e serviram de base para remoção e ou adequação dos equipamentos (ANVISA, 2013; ANVISA, 2001).

\section{CONSTRUÇÃO E APLICAÇÃO DOS EPIs E EPCs}

\section{Protetores faciais - Faceshields}

Baseando-se no modelo criado pelo Projeto Hígia, os protetores faciais foram reproduzidos para suprir a demanda existente na região. Este modelo de baixo custo foi desenvolvido passando por versões distintas até se tornar o mais adequado, sendo validado por diversos profissionais da saúde. O protetor facial, conforme a figura 4, consiste em duas partes: uma tiara, usada para fixar o escudo na altura da testa distante do rosto, e um escudo protetor transparente, que se interpõe à face do usuário e ao ambiente, reduzindo a exposição a gotículas. Inicialmente as tiaras foram feitas por manufatura aditiva, porém, com a crescente demanda da região, passaram a ser feitas por injeção de plástico, tornando a produção mais rápida. Ao mesmo tempo, os escudos foram feitos com folhas de acetato e PETG. Até a data atual, foram doados cerca de 5.500 faceshields para 35 cidades do Rio Grande do Sul. 
Figura 4 - Protetor facial feito por injeção plástica

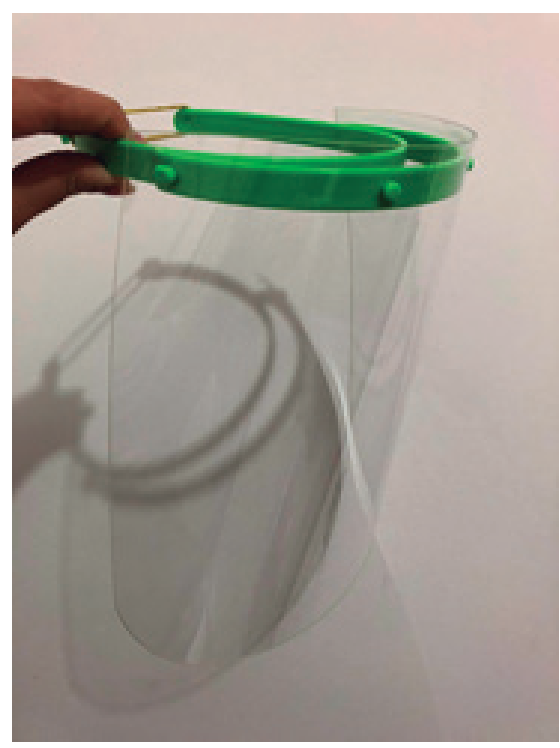

Fonte: Construção do autor

\section{Sistemas de maçanetas handsfree}

Os sistemas de maçaneta handsfree (figura 5A), foram desenvolvidos pois sabe-se que uma das maiores fontes de contaminação é o contato. Nos hospitais há um enorme fluxo de pessoas, sendo elas pacientes, familiares ou trabalhadores, fazendo com que a transmissão do vírus seja alta pelo manuseio das maçanetas das portas com as mãos.

Para diminuir esse contágio, foi criado o dispositivo chamado handsfree - tradução precisa "mãos livres" - a partir de modelos encontrados na internet passando por adaptações, impressos por manufatura aditiva e acoplado as maçanetas. Com isso, as portas são abertas e fechadas utilizando o antebraço, evitando que toquem a superfície com as mãos, conforme a figura 5B. No total, foram doados 55 sistemas de maçanetas para 3 hospitais da cidade de Santa Maria.

Figura 5 - Imagens referentes as maçanetas handsfree: (A) modelo 3D visto no software de impressão dos dispositivos handsfree; (B) Maneira correta de utilização do dispositivo.

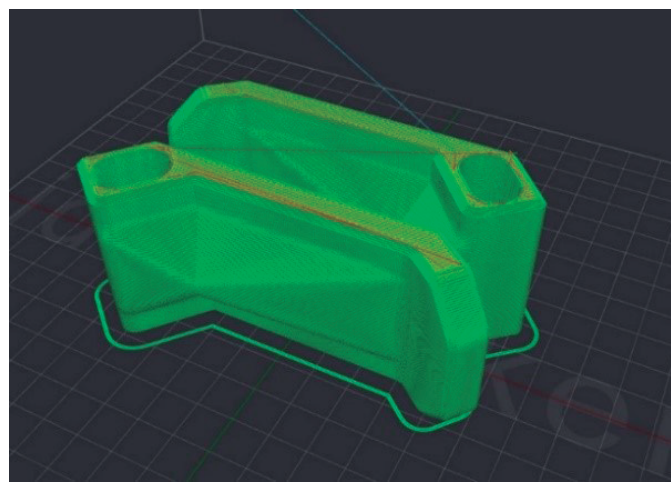

(A)

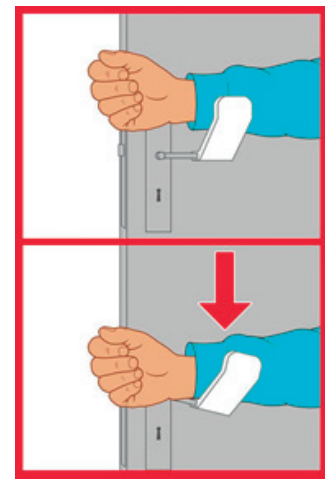

(B)

Fonte: Construção do autor 


\section{Capela de intubação e extubação}

O projeto da capela (figura 6A) surgiu devido à alta exposição dos profissionais da saúde durante os procedimentos de intubação e extubação dos pacientes, mesmo utilizando os EPIs necessários. A intubação consiste na inserção de um tubo - através do nariz ou boca - até a traqueia do paciente, instalando, assim, a ventilação mecânica pulmonar, indicada principalmente em casos de insuficiência respiratória aguda. Para a retirada do tubo, procedimento chamado extubação, é preciso que o paciente faça o movimento de tossir, causando a emissão de aerossóis no ambiente, tornando-o altamente contaminante.

Para prevenir isso, foi criada a capela de intubação, onde é colocada ao redor da cabeça do paciente (figura 6B), protegendo os profissionais em volta. A capela possui uma angulação para visualização apropriada das vias aéreas do paciente pelo médico, e duas aberturas para o encaixe de seus braços. A parte transparente foi feita com policarbonato e possui uma bandeira para encaixe, feito com aço inox, com objetivo de colocar embaixo do paciente e tornar a estrutura mais firme. Para se obter o produto final, foram realizadas medidas, testes e protótipos, além de discussões com os médicos para saber e entender suas necessidades, resultando na doação de 5 capelas para um hospital de Santa Maria.

Figura 6 - Desenhos da construção das peças em ambiente virtual para aprovação das equipes médicas: (A) modelo da capela de intubação e extubação; (B) simulação de um paciente com a capela de intubação

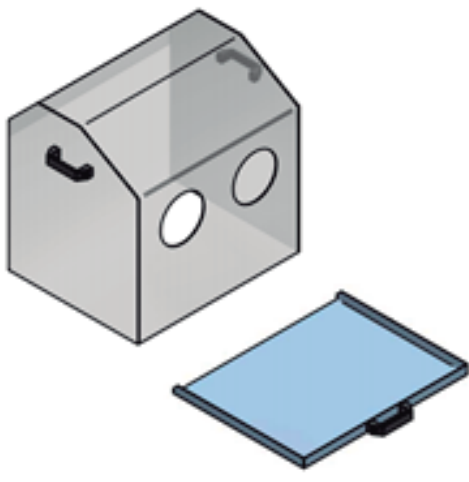

(A)

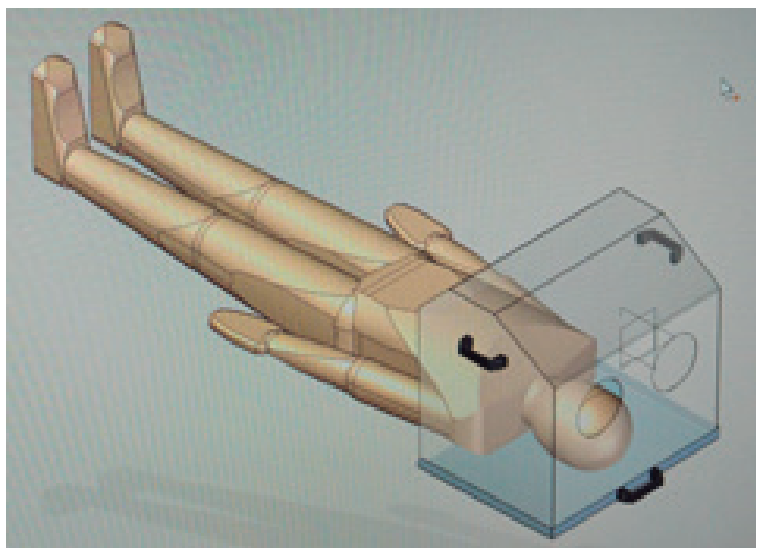

(B)

Fonte: Construção do autor

\section{CONCLUSÃO}

Após a realização de inúmeras visitas ao Hospital Casa de Saúde e Unidade de Pronto-Atendimento de Santa Maria, pode-se concluir o levantamento do parque tecnológico, tornando viável iniciar o Plano de Gerenciamento das Tecnologias em Saúde. Com a criação da Frente de Desenvolvimento Tecnológico à Saúde, foi possível a interação com a comunidade para auxiliar e proteger os 
profissionais da saúde no combate ao Coronavírus. Em ambos trabalhos pode-se perceber a importância da Engenharia Biomédica no contexto hospitalar, trazendo benefícios para o cuidado ao paciente e segurança do profissional da saúde.

\section{REFERÊNCIAS}

ANVISA, Resolução - RDC n⿳ 2, de 25 de janeiro de 2010 - Gerenciamento de Tecnologias em Saúde em Estabelecimentos de Saúde. Brasília, 2010.

ANVISA, Resolução - RDC n 16, de 28 de março de 2013 - Regulamento Técnico de Boas Práticas de Fabricação de Produtos Médicos e Produtos para Diagnóstico de Uso In Vitro e dá outras providências, 2013.

ANVISA, Resolução - RDC n $\mathbf{n}^{\mathbf{0}}$ 185, de 22 de outubro de 2001 - Regulamento que trata do registro, alteração, revalidação e cancelamento do registro de produtos médicos na Agência Nacional de Vigilância Sanitária - ANVISA, 2001.

ANVISA, Resolução - RDC n 356, de 23 de março de 2020 - Regulamento que trata dos requisitos para a fabricação, importação e aquisição de dispositivos médicos identificados como prioritários para uso em serviços de saúde, em virtude da emergência de saúde pública internacional relacionada ao SARS-CoV-2, 2020.

CALIL, S. J.; TEIXEIRA, M. S. Gerenciamento de Manutenção de Equipamentos Hospitalares, volume 11. São Paulo: Faculdade de Saúde Pública da Universidade de São Paulo, 1998. (Série Saúde \& Cidadania).

DYRO, J. F. (Ed.). Clinical engineering handbook. Boston, Massachusetts: Elsevier Academic Press, c2004. xix, 674 p. (Academic Press series in biomedical engineering).

FERRARI, F. COVID-19: Dados Atualizados e sua Relação Com o Sistema Cardiovascular. Arq Bras Cardiol. 2020, v. 114, n. 5, p. 823-826. [online]. Disponível em: https://bit.ly/3n5Msm9. Acesso em: 24 ago. 2020.

MINISTÉRIO DA SAÚDE; Coronavírus: o que você precisa saber? Disponível em: https://coronavirus.saude.gov.br. Acesso em: 24 ago. 2020. 
NEVES, Ursula. Covid-19: Brasil ultrapassa a marca de cem médicos mortos por conta do vírus. Portal PEBMED, 2020. Disponível em: https://bit.ly/2VKHdfo. Acesso em: 22 nov. 2020.

OLIVEIRA, Elida. Brasil tem 30 mortes de profissionais de enfermagem por coronavírus e mais de 4 mil afastados pela doença. G1, 2020. Disponível em: https://glo.bo/36RE4Rt. Acesso em: 22 nov. 2020.

PEREIRA, A. J. F.; GONÇALVES, B. M.; ALMEIDA, R. M. V. R. Produção científica da engenharia biomédica brasileira, junho 2008 - junho 2010. In: CONGRESSO BRASILEIRO DE ENGENHARIA BIOMÉDICA, 2010, Tiradentes. Anais... CBEB, 2010, p. 1077-1080. Disponível em: https://bit.ly/ 3gkzfTL. Acesso em: 19 ago. 2020.

RIBEIRO, A. P. et al. Saúde e segurança de profissionais de saúde no atendimento a pacientes no contexto da pandemia de Covid-19: revisão de literatura. Rev. Bras. Saúde Ocup. [Online], v. 45, 45:e25. São Paulo, 2020. Disponível em: https://bit.ly/3mTZym7. Acesso em: 24 ago. 2020.

TEIXEIRA, C. M. E. et al. Análise comparativa das pandemias COVID-19 e H1N1. Braz. J. Hea. Rev., Curitiba, v. 3, n. 2, p. 3591-3595 mar./apr. 2020. Disponível em: https://bit.ly/31LFcdy. Acesso em: 24 ago. 2020.

WORLDOMETER. COVID-19 Coronavirus pandemic. 1 p. da web. Disponível em: https://www. worldometers.info/coronavirus/. Acesso em: 31 ago. 2020. 